\title{
Avaliação da Aplicabilidade de Técnicas MIC/FT-IR/DSC para a Caracterização de Filmes Multicamadas
}

\author{
Luciano M. Nogueira \\ Instituto Tecnológico de Aeronáutica (ITA) \\ Rita C.L. Dutra, Milton F. Diniz \\ Divisão de Química do Comando-Geral de Tecnologia Aeronáutica, CTA/IAE \\ Marcia Pires, Mônica Evangelista, Fernanda A. Santana, Leandro Tomasi, Priscila dos Santos, Regina Nonemacher
Braskem S.A III
}

Resumo: Técnicas FT-IR de transmissão, reflexão atenuada (ATR), reflexão difusa (DRIFT), microscopia acoplada ao FT-IR (MIC/FT-IR) e detecção fotoacústica (PAS) foram utilizadas para a caracterização de filmes multicamadas. As técnicas ATR, MIC/FT-IR e PAS apresentaram os melhores resultados, provavelmente em função da possibilidade de análise somente da superfície das amostras com cristal (ATR), análise de cada camada da amostra separadamente (MIC/FT-IR) e possibilidade de variação da velocidade de obtenção do espectro (PAS). Dados de análises de microscopia ótica (MO) e calorimetria exploratória diferencial (DSC) foram usados, associados aos dados FT-IR, para a caracterização completa dos materiais.

Palavras-chave: MO, infravermelho com transformada de Fourier (FT-IR), ATR, MIC/FT-IR, DRIFT, PAS, DSC, filmes multicamadas.

\section{Evaluation of MIC/FT-IR/DSC techniques for multilayer films characterization}

Abstract: The transmission, reflection, photoacoustic (PAS) and microscopy FT-IR techniques have been used for characterizing multilayer films. The ATR, microscopy FT-IR and PAS techniques showed better results, probably because it was possible to analyze only the sample surface by ATR, while with MIC/FT-IR each layer of the sample could be studied separately and in PAS the spectra could be obtained at different rates. A complete characterization for the films was achieved by combining with optical microscopy, DSC and FT-IR data.

Keywords: Optical microscopy, DSC, FT-IR, ATR, DRIFT, PAS, multilayer films.

\section{Introdução}

Materiais plásticos, semi-rígidos ou flexíveis, mono ou multicamadas, sob a forma de copos, bandejas, tubos e garrafas, possuem larga aplicação nas indústrias alimentícia, farmacêutica e de cosméticos, entre outras ${ }^{[1]}$. Gradativamente, nas últimas décadas vêm substituindo embalagens convencionais, feitas até então de metais ou vidro, por serem, mais leves, não sofrerem corrosão, alterações em função do pH e principalmente por possuírem um custo menor ${ }^{[2]}$. Materiais multicamadas podem ser obtidos através de laminação ou coextrusão.

Laminados poliméricos são definidos como materiais constituídos por camadas de dois ou mais tipos de polímeros, unidas por um adesivo adicionado na etapa de laminação dos filmes, visando a melhoria quanto à capacidade de conservação, proteção e visual do material final.

Filmes co-extrusados são constituídos de várias camadas, porém são obtidos em uma única etapa de extrusão, onde cada material é alimentado em uma camada diferente. No caso de materiais incompatíveis é usado, no processo de co-extrusão, um adesivo especial (conhecido como adesivo de co-extrusão) para evitar a delaminação das camadas.

Freqüentemente, os plásticos multicamadas utilizados em embalagens são compostos de várias camadas de diferentes materiais, o que permite atingir propriedades, a um custo aceitável $^{[2,3]}$, as quais um plástico monocamada não atingiria por si só ${ }^{[4]}$. Dos materiais mais utilizados nos plásticos multicamadas, podemos citar como exemplo, PE/Al/P; LDPE e EVA, (PET/PE/EVOH/PE), como filmes para embalagem e alimentos.

Existem vários processos para produção de filmes multicamadas $^{[5-6]}$. Diferentes processos e tipos de resina podem afetar significativamente a estrutura química e por conseqüência, as propriedades dos laminados poliméricos ${ }^{[7]}$. Por exemplo, a simples adição de uma camada individual a um filme multicamada EVOH/LPDE pode não ser suficiente para constituir uma nova estrutura de filme multicamada, em função

Autor para correspondência: Rita C.L. Dutra, Divisão de Química do Comando-Geral de Tecnologia Aeronáutica (CTA)/IAE, Pça Mal. do Ar Eduardo Gomes 50, VI. das Acácias, CEP 12228-904, S. José dos Campos, SP, Brasil. E-mail: jrdutra@directnet.com.br 
de problemas relacionados com a adesão entre as camadas ${ }^{[3]}$. Por outro lado, a utilização de PP em filmes multicamadas promoverá uma firmeza do filme, força e uma extensão da temperatura de trabalho ${ }^{[7]}$. Portanto, a caracterização das diferentes camadas constitui uma importante ferramenta para o conhecimento da estrutura química e, conseqüentemente, da adequada aplicação desses materiais.

Estudos são citados ${ }^{[4,8-9]}$ na literatura para caracterização de filmes mulitcamadas. Francis M. Mirabella ${ }^{[4]}$ utilizou técnicas de microscopia óptica, microscopia combinada com FT-IR e outras técnicas auxiliares para a análise qualitativa de estruturas complexas de filmes multicamadas. A microscopia ótica foi utilizada para visualização das camadas presentes no filme. Análises por FT-IR (técnicas de transmissão, ATR e microscopia-FT-IR para identificação das camadas. Também foram utilizadas algumas técnicas complementares como DSC e espectroscopia de ressonância magnética nuclear (NMR) na identificação/caracterização e quantificação dos componentes e determinação da composição de um componente particular do filme multicamada embora, seja mencionado que essas últimas técnicas não são aplicáveis para todos os casos de filmes multicamadas, devido a dificuldade de se ter cada componente fisicamente separado.

Outras metodologias FT-IR, menos convencionais, tais como espectroscopia PAS podem ser aplicadas à caracterização de diferentes materiais ${ }^{[10-11]}$. A caracterização e quantificação por espectroscopia IR de diversos polímeros e suas blendas constitui, também, uma importante linha de pesquisa desenvolvida em nosso Centro de Pesquisa ${ }^{[10,12-15]}$. Entretanto, a combinação de técnicas FT-IR de transmissão, reflexão e PAS não foi ainda utilizada para a caracterização de filmes multicamadas em nossos laboratórios.

Atualmente existem poucos estudos de caracterização de filmes multicamadas, e levando-se em conta a disponibilidade de técnicas FT-IR em nossos laboratórios, nesse trabalho foi feita uma avaliação de técnicas ATR, DRIFT, MIC-FT-IR e PAS (com variação de velocidade de análise) para a caracterização de laminados poliméricos. Técnicas de microscopia e análise térmica foram, também, utilizadas na metodologia desenvolvida para complementar os resultados.

\section{Experimental}

\section{Amostras}

As amostras analisadas foram codificadas como 0400/126IR e 0400/127-IR. A composição desses materiais é desconhecida.

\section{Análise por microscopia}

Análises de microscopia ótica foram realizadas, após preparo das amostras com micrótomo LEICA RM 2145. A identificação do número de camadas presente nos filmes foi realizada utilizando-se um microscópio ótico Leica modelo
DMLM. As imagens obtidas foram tratadas no software Leica Q-Win para determinação da espessura de cada camada.

\section{Análise por FT-IR}

As amostras foram analisadas por FT-IR nos modos transmissão e as superfícies dos filmes foram analisadas por reflexão (ATR, DRIFT) e PAS. Os materiais de referência (calibração) utilizados para as análises por ATR, DRIFT e PAS foram, respectivamente, cristais KRS-5 (45), espelho de calibração e negro de fumo compactado (padrão MTEC).

As análises por transmissão (técnica pastilha $1 / 400 \mathrm{mg}$ $\mathrm{KBr}$ ), DRIFT, ATR (cristal KRS-5 e Ge) e PAS foram conduzidas com o espectrômetro FT-IR SPECTRUM 2000 PERKINELMER.

Para análise FT-IR por transmissão foram utilizadas as seguintes condições: resolução de $4 \mathrm{~cm}^{-1}$; ganho 1; 4000 a $400 \mathrm{~cm}^{-1}, 40$ varreduras. As análises por ATR (KRS-5) foram feitas na região de 4000 a $400 \mathrm{~cm}^{-1}$ e de 4000 a $700 \mathrm{~cm}^{-1}$ para o cristal de gerânio, $10 \%$ de energia, 40 varreduras, sendo que a amostra foi colocada sobre os dois lados do cristal. As análises por DRIFT foram feitas na região de 4000 a $400 \mathrm{~cm}^{-1}, 30 \%$ de energia. Para a análise PAS, as condições foram: resolução 4, velocidade 0,05 e $0,2 \mathrm{~cm} / \mathrm{s}, 4000$ a $400 \mathrm{~cm}^{-1}, 32$ - 128 varreduras e ganho 6 no amplificador. Essas análises foram realizadas nos laboratórios do CTA. As análises MIC/FT-IR foram conduzidas no Centro de tecnologia da Braskem S.A, em equipamento Nicolet 710 e Nicolet 710/ microscópio ótico Nicolet Continum respectivamente. Para as análises foram utilizadas as seguintes condições: $4 \mathrm{~cm}^{-1}$; ganho 1; 4000 a $400 \mathrm{~cm}^{-1}, 200$ varreduras.

\section{Análise DSC}

As análises DSC foram realizadas em equipamento DSC TA Instruments, modelo 2910 no Centro de tecnologia da Braskem S.A. Aproximadamente 5,5 g de amostra foram aquecidas a $200{ }^{\circ} \mathrm{C}$ a uma taxa de $10{ }^{\circ} \mathrm{C} / \mathrm{min}$. Após resfriamento, a amostra passou por novo aquecimento a $300{ }^{\circ} \mathrm{C}$, a uma taxa de $10{ }^{\circ} \mathrm{C} / \mathrm{min}$, para determinação da temperatura de fusão.

\section{Resultados e Discussão}

\section{Análise de microscopia ótica}

Por meio da análise de MO foi possível observar na amostra 0400/126-IR, a presença de 3 camadas, com aproximadamente $150 \mu \mathrm{m}$ de espessura média no total. A análise MO da amostra 0400/127-IR mostrou a presença de 5 camadas, com aproximadamente $50 \mu \mathrm{m}$ de espessura no total (Figura 1).

\section{Caracterização por FT-IR da amostra 0400/126-IR}

\section{Análise por FT-IR de transmissão}

As absorções médias e intensas observadas no espectro FT-IR de transmissão (pastilha de KBr) da amostra 0400/126IR foram escolhidas para caracterizar os grupos funcionais do 


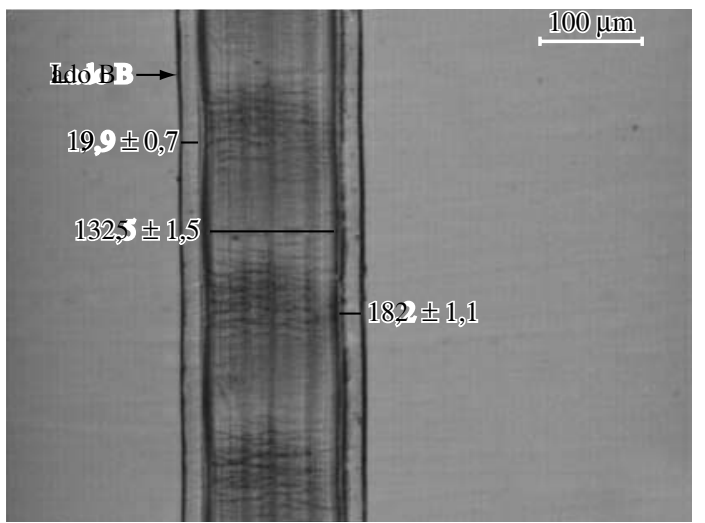

(a)

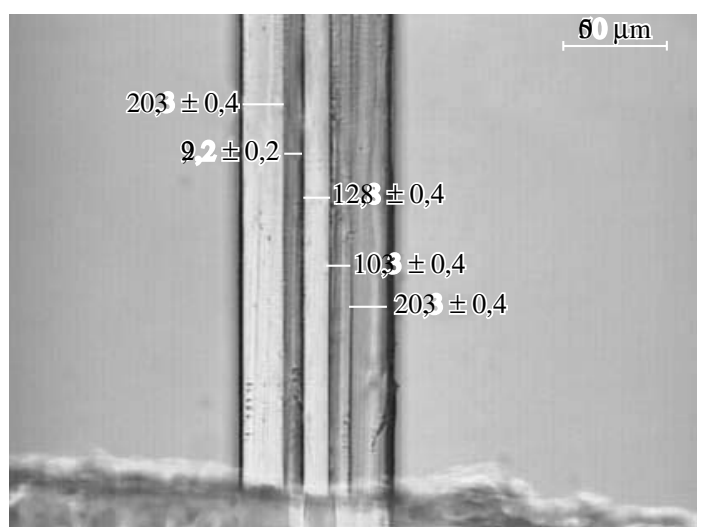

(b)

Figura 1. Micrografias óticas das amostras a) 0400/126-IR; e b) 0400/127-IR. (Espessura média em $\mu \mathrm{m}$ ).

composto, em acordo com a literatura ${ }^{[16-17]}$. O mesmo foi feito para a outra amostra.

O conjunto de absorções $\left(\mathrm{cm}^{-1}\right)$, associado ao seu formato, em 1724 ( $\mathrm{vC}=\mathrm{O}), 1249,1165,1111$ e 1042(v C-O) e $720\left(\mathrm{CCH}_{2}\right)$ sugere a presença de polímero à base de éster, provavelmente alifático, apresentando cadeia linear com mais de 3 átomos de $\mathrm{CH}_{2}$, devido a presença da banda intensa em $720 \mathrm{~cm}^{-1}$. Essa banda pode, também, sugerir a presença de uma poliolefina ${ }^{[16]}$ de estrutura similar a encontrada em polietileno (PE). Também foi observada a absorção em $1165 \mathrm{~cm}^{-1}$ que pode sugerir a presença de polipropileno (PP). A banda em $3500 \mathrm{~cm}^{-1}$ pode ser atribuída a $(\mathrm{VO}-\mathrm{H})$, devido à presença de umidade na pastilha de $\mathrm{KBr}$,

A indicação de diferentes compostos (PE, PP, éster alifático) está de acordo com as características da técnica de transmissão, que revela a composição como um todo ${ }^{[16]}$.

\section{Análise por FT-IR/ATR $x$ transmissão}

Na Figura 2 estão incluídos os espectros obtidos por ATR (KRS-5) de ambos os lados da amostra (lado A e lado B) em comparação com o espectro de transmissão. É possível observar que os espectros são diferentes, sugerindo a presença de estruturas distintas nas diferentes camadas do filme. O espectro III, obtido por transmissão, conforme discutido, representa as absorções de um poliéster alifático, podendo ainda indicar a presença de poliolefina.
No espectro obtido por ATR (KRS-5) I - lado A, foram observadas bandas $\left(\mathrm{cm}^{-1}\right)$ características ${ }^{[17-18]} \mathrm{em:} 2949$ $\left(v_{\mathrm{a}} \mathrm{CH}_{3}\right), 2920\left(v_{\mathrm{a}} \mathrm{CH}_{2}\right), 2866\left(v_{\mathrm{s}} \mathrm{CH}_{3}\right), 2852\left(v_{\mathrm{s}} \mathrm{CH} 2\right)$, $1454\left(\delta \mathrm{a} \mathrm{CH}_{3}\right.$ e $\left.\delta_{\mathrm{s}}^{\mathrm{a}} \mathrm{CH}_{2}\right), 1376\left(\delta_{\mathrm{s}} \mathrm{CH}_{3}\right), 1166\left(\delta \mathrm{a} \mathrm{CH}_{3}\right)$, $997(\omega \mathrm{C}=\mathrm{C}$ vinil $)$ ou $\left(\delta \mathrm{CH} \rho \mathrm{CH}_{3}, \varpi \mathrm{CH} 2\right), 972(\omega \mathrm{C}=\mathrm{C}$ trans) ou ( $\left.\delta \mathrm{CH}_{3}, v \mathrm{C}-\mathrm{C}\right), 721\left(\rho \mathrm{CH}_{2}\right)$, podendo tratar-se de polipropileno (PP) e PE As bandas que diferenciam PP de PE são, principalmente, 1166, 997 e $972 \mathrm{~cm}^{-1}$ (PP) e $721 \mathrm{~cm}^{-1}$ (PE). O espectro ATR (KRS-5) II - lado B apresentou bandas $\left(\mathrm{cm}^{-1}\right)$ características em $1719(\mathrm{~V} \mathrm{C}=\mathrm{O}), 1246$ e $1039 \mathrm{~cm}^{-1}$ ( $\vee \mathrm{C}-\mathrm{O})$, podendo tratar-se de poliéster alifático ${ }^{[17]}$.

O conjunto de dados ATR sugere, então, que uma das superfícies da amostra, identificada como lado A, é constituída de poliolefinas enquanto que a outra superfície é constituída de poliéster, compostos encontrados em filmes multicamadas usados em diferentes formas de embalagens ${ }^{[9]}$

\section{Análise por FT-IR/DRIFT $x$ transmissão}

Na Figura 3 estão incluídos os espectros obtidos das superfícies A e B por DRIFT em comparação com espectro de transmissão da amostra 0400/126-IR.

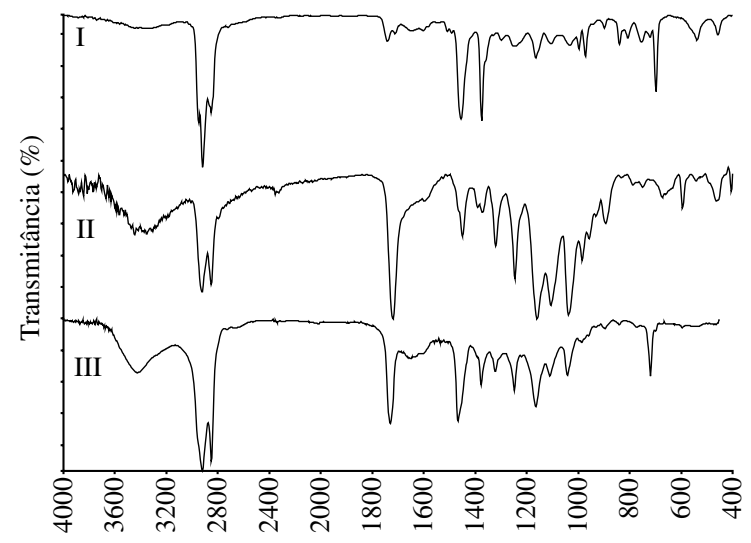

Número de ondas $\left(\mathrm{cm}^{-1}\right)$

Figura 2. Espectros FT-IR da amostra 0400/126-IR - I (ATR/KRS-5-lado A); II (ATR/KRS-5-lado B); e III (transmissão).

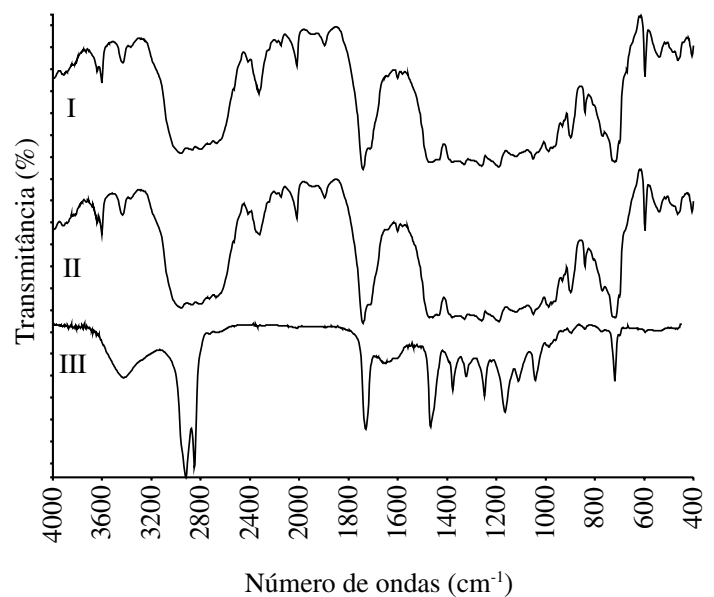

Figura 3. Espectros FT-IR da amostra 0400/126-IR - I (DRIFT- lado A); II (DRIFT - lado B); e III (transmissão). 
É possível observar que os espectros são bastante similares, em relação aos números de onda, diferindo somente na espessura do filme analisado. Estes resultados estão de acordo com o esperado pela técnica, já que, em um experimento DRIFT, reflexões de superfície e do interior da amostra são medidas. Nos espectros DRIFT dos lados A e B da amostra (espectros I e II), observa-se, como no espectro obtido por transmissão, a presença de uma banda em torno de $1710 \mathrm{~cm}^{-1}$, que está na região de carbonila. Além da banda a $1710 \mathrm{~cm}^{-1}$, também foi observado uma banda em $1743 \mathrm{~cm}^{-1}$, a qual deve estar associada à presença de mais de um tipo de poliéster.

\section{Análise por MIC/FT-IR}

A identificação da camada central da amostra foi realizada por MIC/FT-IR, onde é possível focar o feixe de infravermelho em um ponto específico da amostra e obter o espectro referente ao ponto escolhido. $\mathrm{O}$ espectro obtido (Figura 4) apresentou bandas em 1740 e $1240 \mathrm{~cm}^{-1}$, atribuídas à presença, respectivamente, de grupos $\mathrm{C}=\mathrm{O}$ e $\mathrm{C}-\mathrm{O}$ de EVA. A observação de banda em $1740 \mathrm{~cm}^{-1}$ está de acordo com os dados DRIFT.

\section{Análise FT-IR/PAS}

Nesse trabalho, as diferentes velocidades de modulação da freqüência utilizadas na análise PAS foram utilizadas para auxiliar a identificação da composição de cada camada da amostra.

Na Figura 5 estão incluídos os espectros obtidos pela técnica PAS (com diferentes velocidades) do lado A (I e II) e do lado B (III e IV) da amostra do filme polimérico 0400/126-IR.

No espectro I, é possível observar que utilizando-se a velocidade mais lenta, ou seja, $0,05 \mathrm{~cm} / \mathrm{s}$, as bandas do PP, já discutidas nos tópicos anteriores, são melhor visualizadas, sugerindo que esse polímero encontra-se numa camada mais interna de acordo com as características da técnica.

Com o uso da velocidade $0,2 \mathrm{~cm} / \mathrm{s}$, no espectro II, embora as absorções de PP possam ser visualizadas, é possível observar, banda mais intensa em torno de $720 \mathrm{~cm}^{-1}$, sugerindo a presença de polietileno em camada mais superficial.

No espectro III, é possível observar que, utilizando-se a velocidade $0,05 \mathrm{~cm} / \mathrm{s}$, as bandas de $P E$ são visualizadas, sugerindo que esse polímero encontra-se numa camada mais interna. Com velocidade mais alta, $0,2 \mathrm{~cm} / \mathrm{s}$, no espectro IV, absorções de poliéster alifático são visualizadas, sugerindo que esse componente esteja em camada mais superficial.

É possível sugerir, pela análise PAS, que o lado A da amostra 0400/126-IR é constituído de PP/PE e o lado B por poliéster, possuindo uma camada de PE mais interna. É interessante observar que a visualização de absorções de poliéster alifático no lado B da amostra 0400/126-IR em camada mais superficial, indicada pela análise PAS, está em concordância com a análise ATR.

\section{Caracterização por FT-IR da amostra 0400/127-IR}

\section{Análise por FT-IR de transmissão}

O conjunto de absorções $\left(\mathrm{cm}^{-1}\right)$, associado ao seu formato, em $3310(v \mathrm{OH} / v \mathrm{NH}), 1740(v \mathrm{C}=\mathrm{O}), 1642\left(v \mathrm{C}=\mathrm{O} / \delta \mathrm{NH}_{2}\right)$, $1546(\delta \mathrm{NH}+v \mathrm{C}-\mathrm{N}), 1241,1120,1083,1020(v \mathrm{C}-\mathrm{O})$ e $720\left(\mathrm{CCH}_{2}\right)$ sugere a presença de polímeros com ligações $\mathrm{OH}$ e $\mathrm{C}-\mathrm{O}$, características de poliálcoois, tipo EVOH; ligações $\mathrm{C}=\mathrm{O}, \mathrm{C}-\mathrm{O}$ e $\mathrm{CH}_{2}$ encontradas nos poliésteres tipo acetato (EVA), ligações $\mathrm{C}+\mathrm{O}$ e $\mathrm{NH}_{2}$, características de poliamidas e ligações $\mathrm{CH}_{2}$ poliolefinas, tais como polietileno.

\section{Análise por FT-IR/ATR $x$ transmissão}

Na Figura 6 estão incluídos os espectros obtidos por ATR (KRS-5) das superfícies A e B e transmissão da amostra de laminado polimérico 0400/127-IR. É possível observar que tanto o lado A quanto o lado B dessa amostra, apresentam absorções de EVA.

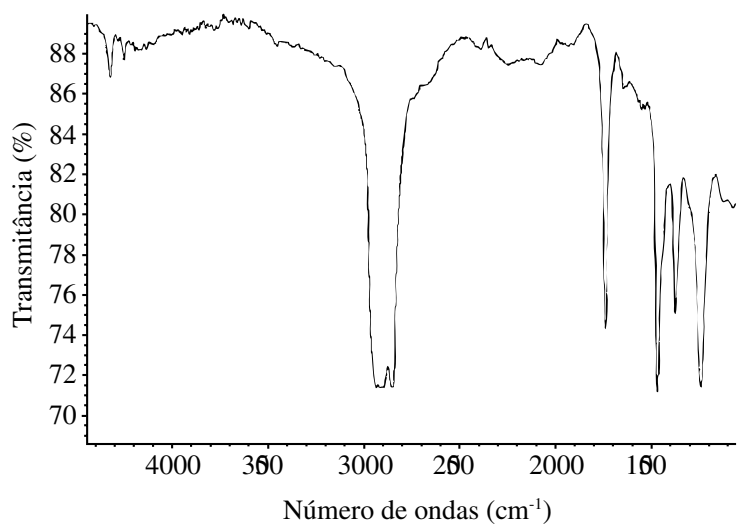

Figura 4. Espectro MIC/FT-IR da amostra 0400/126-IR - camada central.

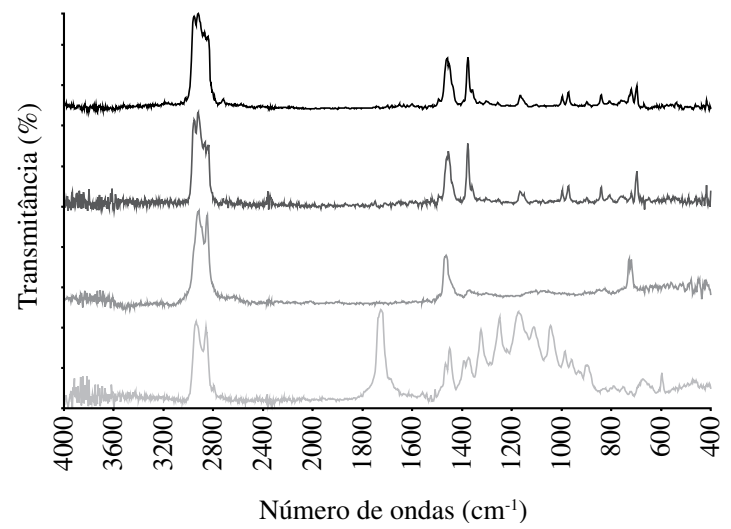

Figura 5. Espectros FT-IR PAS da amostra 0400/126-IR: I (lado A $0,05 \mathrm{~cm} / \mathrm{s}$ ); II (lado A - 0,2 cm/s); III (lado B - 0,05 cm/s); e IV (lado B $0,2 \mathrm{~cm} / \mathrm{s})$. 


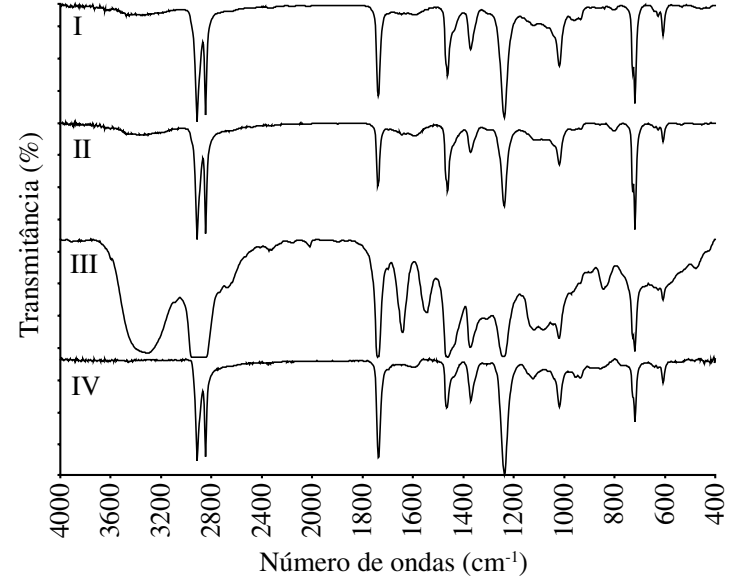

Figura 6. Espectros FT-IR da amostra 0400/127-IR - I (ATR/KRS-5 lado A); II (ATR/KRS-5 - lado B); III (transmissão); e IV (EVA).

A amostra foi também analisada por ATR, utilizando-se o cristal de Ge. A comparação dos dados obtidos por ATR (KRS-5) e ATR (Ge) são mostrados na Figura 7.

É interessante observar que ambos os lados da amostra (lado A e B), analisados por ATR (KRS-5) (espectros I e III) sugerem a presença de EVA, e por ATR $(\mathrm{Ge})$ (espectros II e IV), absorções em torno de 3300, 1650 e $1100 \mathrm{~cm}^{-1}$, foram observadas podendo ser associadas a polímeros contendo grupos álcool e/ou amida. Provavelmente, essa diferença pode estar associada ao grau de profundidade de amostragem, quando se utilizam cristais diferentes.

O conjunto de dados ATR sugere, então, que ambas as superfícies (lado A e B) sejam constituídos por polímeros à base de éster, tipo EVA, com possibilidade de compostos à base de álcool (EVOH) e/ou poliamida estarem em diferentes camadas.

\section{Análise por FT-IR/DRIFT $x$ transmissão}

Desde que a análise DRIFT, realizada em ambos os lados da amostra 0400/127-IR, mostrou o mesmo resultado que o obtido por transmissão, ou seja, indicou a presença de

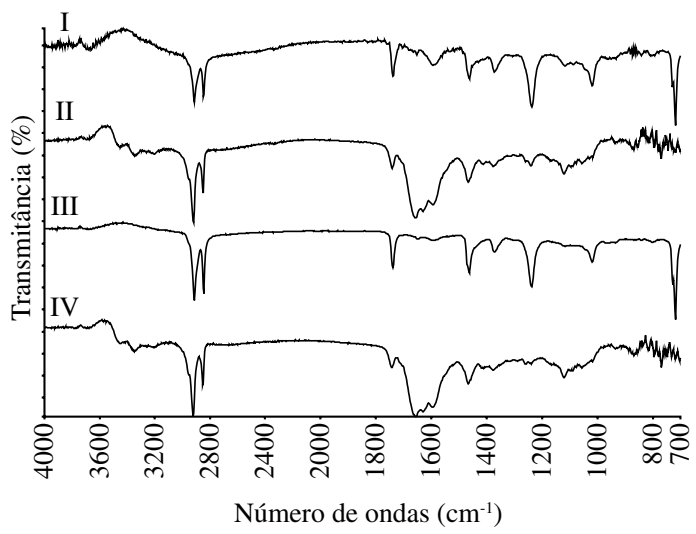

Figura 7. Espectros ATR da amostra 0400/127-IR (lado A) - I (ATR/KRS-5); II (ATR/Ge) (lado B); - III (ATR/KRS-5); e IV (ATR/Ge).
EVOH, EVA, poliamida e PE, os espectros não foram incluídos nessa discussão.

\section{Análise FT-IR/PAS}

Na Figura 8 estão incluídos os espectros obtidos pela técnica PAS analisando-se uma das superfícies do filme (lado A) utilizando-se diferentes velocidades. Utilizando-se a velocidade mais rápida, ou seja, $0,2 \mathrm{~cm} / \mathrm{s}$, é possível observar as bandas do EVA, sugerindo que este polímero se encontra na camada mais superficial da amostra.

Utilizando-se a menor velocidade, $0,05 \mathrm{~cm} / \mathrm{s}$, são observadas absorções em torno de 33001650 e $1100 \mathrm{~cm}^{-1}$ que podem sugerir a presença de EVOH e poliamida em camadas mais internas da amostra.

Na Figura 9 estão incluídos os espectros obtidos pela técnica PAS analisando-se a outra superfície do filme (lado B) utilizando-se diferentes velocidades. É possível observar que, utilizando-se a velocidade $0,05 \mathrm{~cm} / \mathrm{s}$, as bandas de $\mathrm{EVOH}$ e poliamida são melhor visualizadas, sugerindo que esse polímeros encontram-se em camadas mais internas. Com velocidade mais alta, $0,2 \mathrm{~cm} / \mathrm{s}$, absorções de EVA são melhor visu-

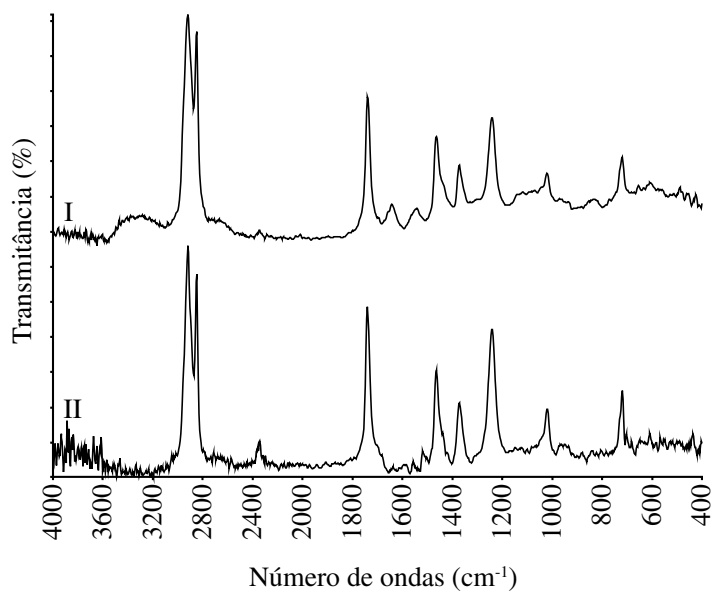

Figura 8. Espectros FT-IR PAS da amostra 0400/127-IR: I (lado A-0,05 cm/s); e II (lado A- $0,2 \mathrm{~cm} / \mathrm{s}$ ).

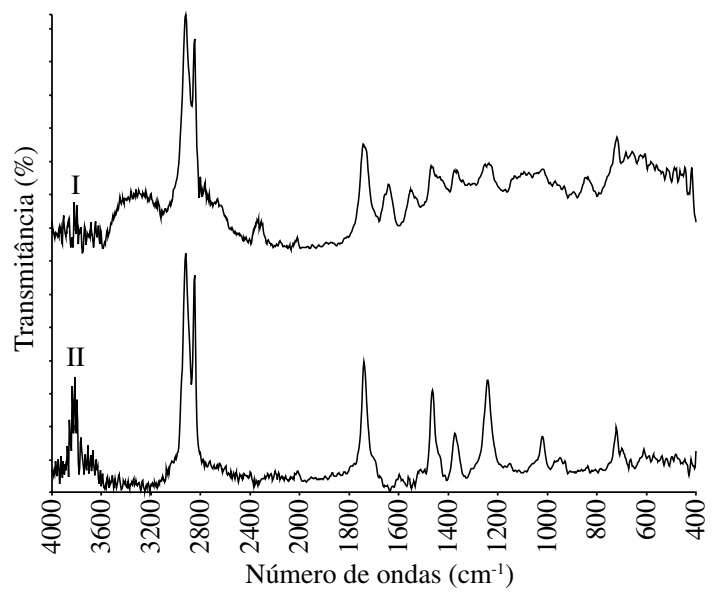

Figura 9. Espectros FT-IR PAS da amostra 0400/127-IR: I (lado B $0,05 \mathrm{~cm} / \mathrm{s}$ ); e II (lado B- $0,2 \mathrm{~cm} / \mathrm{s}$ ). 
alizadas, sugerindo que este polímero se encontra na camada mais superficial da amostra.

\section{Análise por MIC/FT-IR}

Devido ao grande número de camadas e a complexidade da estrutura foi realizada uma análise por MIC/FT-IR, onde é possível visualizar e identificar cada camada presente no filme. A Figura 10 mostra o comparativo dos espectros obtidos durante a varredura em todas as camadas do filme. A identificação de cada camada foi realizada, comparando-se

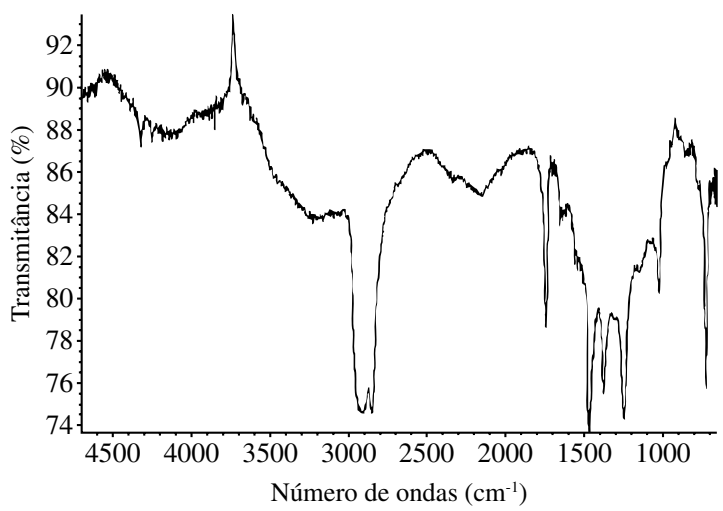

(a)

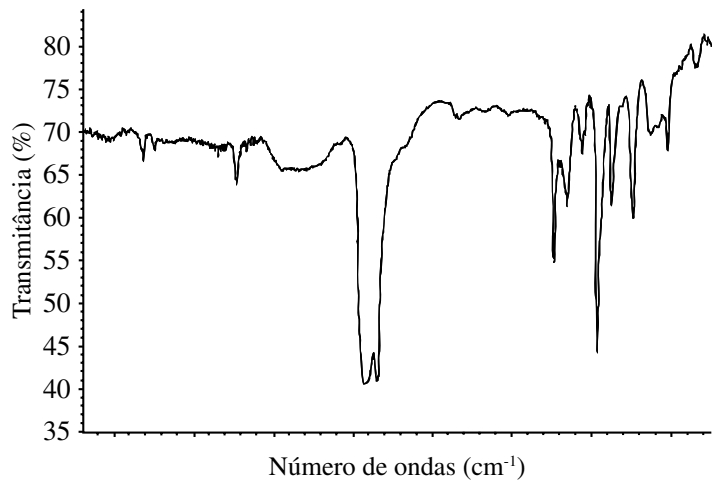

(b)

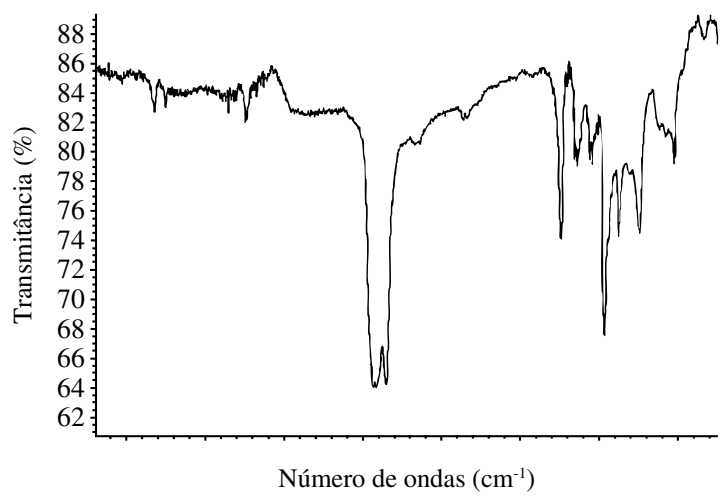

(c)

Figura 10. Espectros MIC/FT-IR da amostra 0400/127-IR: Pontos 1 (espectro a); 3 (espectro b); e 4 (espectro c). todos os espectros separadamente com espectros do banco de dados.

Foram analisados vários pontos diferentes ao longo da espessura da amostra. Os espectros obtidos mostraram a presença de EVA, Poliamida, EVOH, Poliamida e EVA na seqüência da varredura como comentado abaixo.

- Espectro do ponto 1: apresentou bandas características de EVA (espectro a);

- Espectro do ponto 2: apresentou bandas de EVA. Devido a pequena espessura da camada foram observadas as bandas de absorção da camada lateral;

- Espectro do ponto 3: além das bandas de EVA também foram observadas bandas características de EVOH. Devido a espessura da camada foram observadas as bandas de absorção da camada lateral (espectro b);

- Espectro do ponto 4: além das bandas de EVA também foram observadas bandas características de Poliamida. Devido a espessura da camada foram observadas as bandas de absorção da camada lateral (espectro c); e

- Espectro do ponto 5: além das bandas de EVA também foram observadas bandas características de Poliamida. Devido a espessura da camada foram observadas as bandas de absorção da camada lateral.

Os resultados obtidos por MIC/FTIR estão de acordo com os resultados obtidos por PAS, onde foi observada a presença de EVA nas superfícies do filme e poliamida e EVOH em camadas mais internas.

\section{Análise DSC}

A curva DSC da amostra 0400/126-IR apresentou picos de fusão à $92{ }^{\circ} \mathrm{C}$ com entalpia de $59 \mathrm{~J} / \mathrm{g}$; à $130{ }^{\circ} \mathrm{C}$ com entalpia de $1 \mathrm{~J} / \mathrm{g}$ e à $202{ }^{\circ} \mathrm{C}$ com entalpia de $2 \mathrm{~J} / \mathrm{g}$, confirmando a presença de EVA + PEAD + Poliéster. (Figura 11a).

A curva DSC da amostra 0400/127-IR apresentou picos de fusão à 95 e à $121^{\circ} \mathrm{C}$, associados a uma entalpia de $54 \mathrm{~J} / \mathrm{g}$ e à $147{ }^{\circ} \mathrm{C}$ com entalpia de $8 \mathrm{~J} / \mathrm{g}$, indicando tratar-se provavelmente de EVA + PEBDL + EVOH. Apresentou ainda, em sua primeira fusão (Tm1), picos à 193 e à $209{ }^{\circ} \mathrm{C}$ de pouca intensidade, indicando possível presença de Poliamida na estrutura (Figura 11b).

\section{Análise DSC/Associação Dados IR}

Para complementar os resultados obtidos foi realizada uma delaminação da amostra com ácido fórmico, o qual dissolve a maioria das poliamidas. Após a delaminação, as camadas externas foram caracterizadas.

O espectro FT-IR de ambas as camadas apresentou bandas características de EVA. De acordo com resultados anteriormente obtidos.

A curva de DSC apresentou picos de fusão em 93 e $121{ }^{\circ} \mathrm{C}$ com associados a uma entalpia de aproximada- 


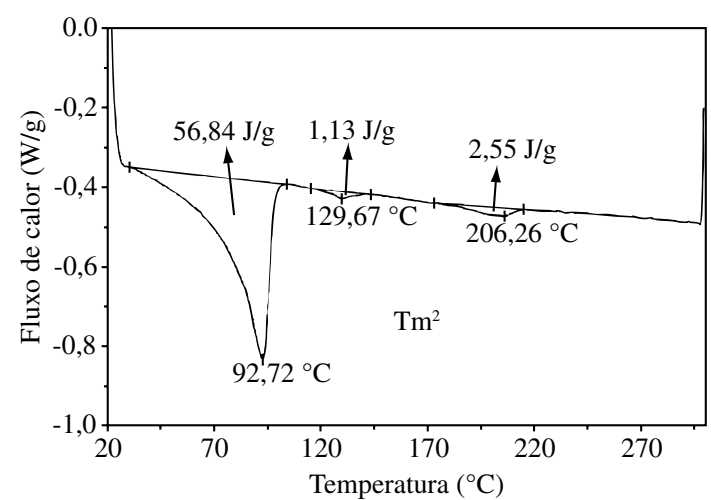

(a)

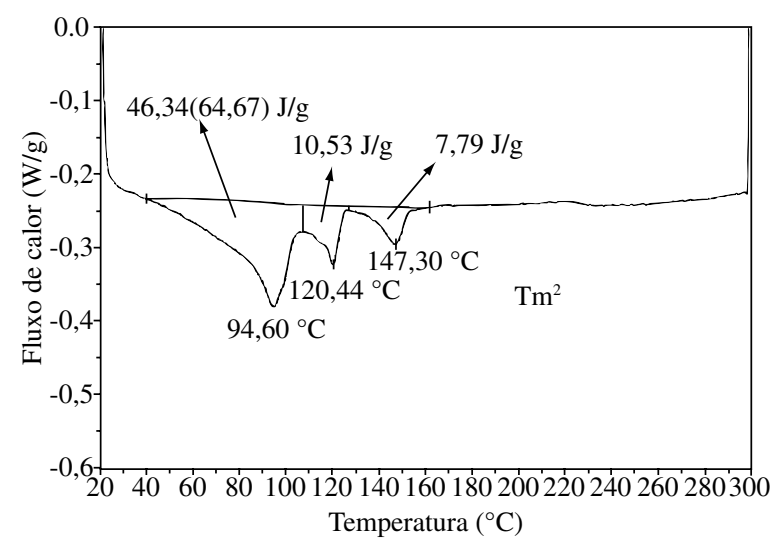

(b)

Figura 11. Curvas DSC: (a) Amostra 0400/126-IR; e (b) Amostra 0400/127-IR. (Exo Up, Universal V4.0 CTA).

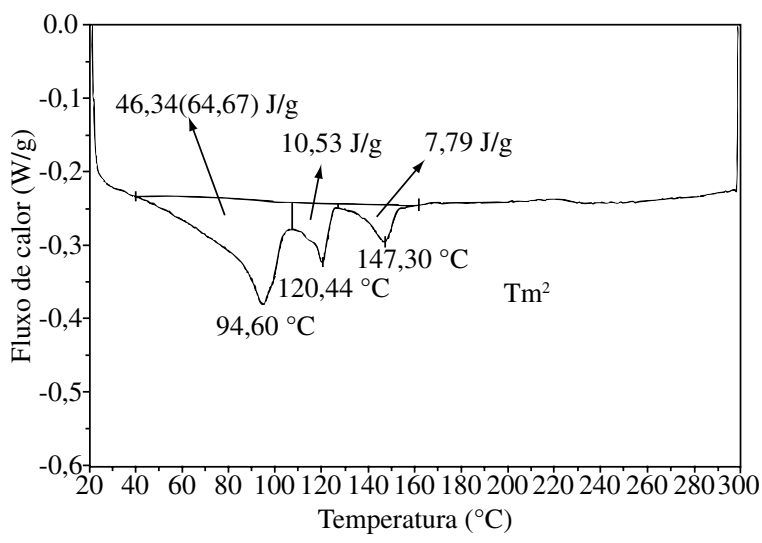

(a)

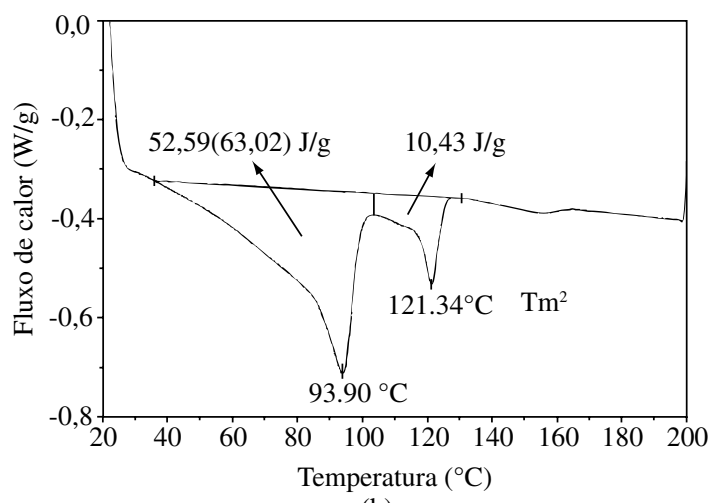

(b)

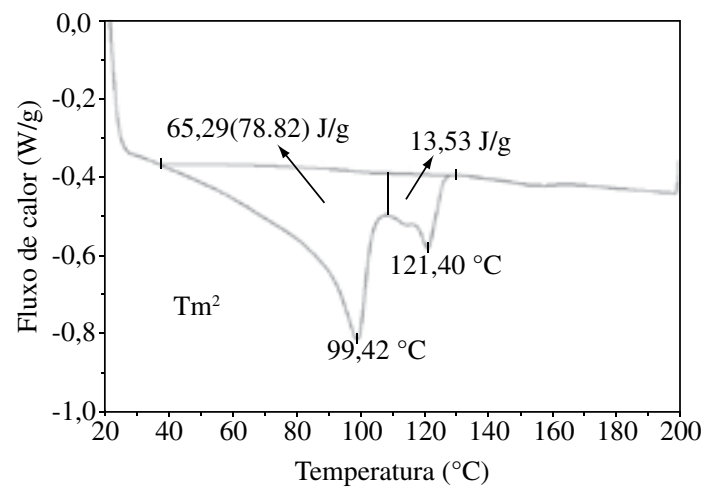

(c)

Figura 12. Curva DSC da amostra 0400/127-IR a) inteira; b); camada A; e c) camada B. (Exo Up, Universal V4.0 CTA). mente $53 \mathrm{~J} / \mathrm{g}$, para a camada $\mathrm{A}$, picos de fusão em 99 e $121{ }^{\circ} \mathrm{C}$ com associados a uma entalpia de aproximadamente $79 \mathrm{~J} / \mathrm{g}$, para a camada B. Os resultados mostram que as camadas externas da amostra são constituídas de EVA + PELBD (Figura 12).

\section{Conclusão}

As técnicas FT-IR ATR, MIC/FT-IR e PAS mostraram os melhores resultados para identificação das camadas, indicadas pela microscopia, provavelmente em função de permitirem variações das condições experimentais, prisma na análise ATR, análise das camadas separadamente (MIC/FT-IR) e velocidade de obtenção do espectro na análise PAS. A análise DSC confirmou, basicamente, os dados FT-IR. Portanto, a aplicação de técnicas MIC/FT-IR/DSC mostrou-se adequada para os materiais analisados, indicando que:

Amostra 0400/126-IR é constituída de 5 camadas sendo observadas por microscopia, somente 3 . Os resultados FTIR e DSC mostram que as camadas contêm PE, PP, EVA e poliéster.

Estrutura proposta: (lado A) Poliéster alifático/PE/ EVA/PE+ PP/ PE +PP (lado B);

Outra proposta de estrutura seria: (lado A) Poliéster alifático/PE/ EVA + PE /PE + PP/ PE +PP (lado B). As camadas PE e PE + PP (mais internas) não foram observadas por Microscopia ótica provavelmente devido à limitação da técnica, indicando tratarem-se de camadas muito finas.

A amostra 0400/127-IR é constituída de 5 camadas contendo EVA, PE, Poliamida e EVOH.

Estrutura proposta: EVA+PE/Poliamida/EVOH/Poliamida/EVA +PE.

Diante dos resultados, conclui-se que a metodologia desenvolvida, dentro das limitações das técnicas, pode ser aplicada para a caracterização de filmes multicamadas.

\section{Referências Bibliográficas}

1. Goulas, A. E.; Riganakos, K. A. \& Kontominas, M. G. Radiation Physics and Chemstry, 69, p.411(2004). 
2. Miltz, J.; Raz, I. \& Passy, N. - Department of Food Engineering and Biotechnology, 28, p.442 (1995).

3. Zhang, X. M.; Ajji, A. \& Marie, V. J. - Polymer, 42. p.8179 (2001).

4. Mirabella, F. M. - Polymer Engineering And Science, Mid May, 26, p.605, (1986).

5. Ezquerro, O.; Pons, B \& Tena, M. T. - Direct - Journal of Chromatography, 985, p.247, (2003).

6. Larena, A.; Millán, F.; Pérez, G. \& Pinto, G. - Apllied Surface Science, 187, p.339, (2002).

7. Zhang, X. M. \& Ajji, A. - Polymer, 46, p.3385, (2005).

8. Irudayaraj, J. \& Yang, H. - Journal of Food Engineering, 55, p.25, (2002).

9. Bubeck, C - Polymer Journal, 23, p. 603, (1991).

10. Mattos, E. C.; Viganó, I.; Dutra, R. C. L.; Diniz, M. F. \& Iha, K-Quim. - Nova, 25, p.722. (2002).

11. Pandey,G. C. \& Kulshreshtha, A. K.- Process Control and Quality, 4, p.109, (1993).

12. Takahashi, M. F. K.; Dutra, R. C. L.; Diniz, M. F. \& Polito, W. L. - Revista Polímeros: Ciência e Tecnologia; 6, p.45, (1996).
13. Dutra, R. C. L. \& Soares, B. G. - Polymer Bulletin 41, p.61, (1998).

14. Evora, C. M.; Gonçalez, O. L.; Dutra, R. C. L.; Diniz, M. F.; Wiebeck, H. \& Silva, L. G. A. - Polímeros Ciência e Tecnologia, 12, p.60, (2002).

15. Dutra, R. C. L.; Diniz, M. F.; Ribeiro, A. P.; Lourenço, V. L.; Cassu, S. N. \& Azevedo, M. F. P - Polímeros Ciência e Tecnologia, 14, p.334, (2004).

16. Smith, A. L. - "Applied Infrared Spectroscopy”, John Wiley \& Sons, New York, (1979).

17. Urbanski et al. - "Handbook of analysis of synthetic polymers and plastics" John Wiley \& Sons, New York, (1977)

18. Andreassem, E. - Polypropylene: Na A - Z Reference, edited by J. Karger-Kocsis Kluwer Publischers, Dordrecht, (1999).

Enviado: 20/11/06

Reenviado: 14/03/07

Aceito: $22 / 03 / 07$ 